\title{
Optimizer in a Box: Simple Economic Optimization of Power Dispatch in Small Microgrids using OPC-UA
}

\author{
Roberto Alexandre Dias \\ IFSC \\ Av. Mauro Ramos, 950 \\ Centro - Florianópolis, Brasil
}

\author{
Gregory das Chagas Gomes \\ IFSC \\ Av. Mauro Ramos, 950 \\ Centro - Florianópolis, Brasil
}

\author{
Marcelo Lobo Heldwin \\ IFSC \\ Campus Reitor João David Ferreira \\ Limas - Trindade, Florianópolis - \\ SC
}

\begin{abstract}
In this paper a simple approach to is presented to economically optimize the power dispatch in a small microgrids environment. The optimization is modeled as a linear program problem and was solved by the Gnu Linear Program Tool Kit (GLPK). The converters and inverters that compose the microgrid use the OPC-UA protocol over Ethernet network to exchange data. The main contribution of this paper is the development of a simple optimizer system and friendly communication framework, compatible with small microgrids using renewable energy sources, like green data-centers and home environments to minimize the energy cost subjected to power limits of energy sources and loads demands.
\end{abstract}

\section{Keywords}

Microgrids, Linear Programming, OPC-UA, Simplex

\section{INTRODUCTION}

According to [1], the increase in the electricity price and efforts for fossil fuels consumption reduction has placed Smartgrids technologies in evidence worldwide, especially in the field of distributed electricity generation. The implementation of small distributed generation arrays with energy resources that provide autonomy, called "microgrid", has been widely promoted by the governments of several countries, especially in Europe, to mitigate the greenhouse effect. In Brazil, the regulatory frameworks of distributed generation are in the beginning of their establishment. The basis for such systems lies on the integration of energy and information networks to form automated, secure, reliable and efficient energy supply systems. The microgrid control, supervision and automation functions provide the means Preprint submitted to Computers and Industrial Engineering February 29, 2016 to achieve those objectives. Thus, the coordination of small scale microgrids operated by people who are not specialized in energy automation systems, like IT teams in a data center installation or home microgrid systems proprietary, demands a simple, robust, secure and low cost solutions. Several optimization approaches applied to microgrids economic minimization were found in literature, but with complex implementation. The communication infrastructure used in electrical system automation, like substations, is very complex and very difficult to integrate with the Internet. In this article, a simple economic energy dispatch optimizer system is proposed and it's called "Optimizer in a Box", running in a personal computer and low cost embedded systems. The communication infrastructure used in this paper is compatible with de IIoT (Industrial Internet of Things) World Economic Forum (2015) - using the new protocol OPC UA (Ole for Process Control Unified Architecture) [9].
This protocol facilitates the applications integration and devices interoperability.

Several papers discuss the use of middleware frameworks to manage and interoperate smartgrids. An overview about the benefits of IEC 61850 is presented in [10].

In [11] the authors propose an OPC UA middleware optimization for energy automation integrated to IEC 61850 In this article a Websocket maps OPC UA to IEC 61850 client and server. The use of this approach favors the interoperation across internet for substation automation.

In [12] it was proposed to replace MMS (Manufacturing Message Specification) middleware with RESTful web services in vertical communications on IEC 61850 to Facilitating a Generic Communication Interface to Distributed Energy Resources.

In both papers, the complexity, inter-operation and security issues on IEC 61850 are target. The use of an SOA enabled middleware like OPC UA for energy systems automation is promissory.

Some papers, like [13], address the microgrid optimization problem. In this paper a MILP (MIxed Integer Linear Program) approach was used to solve Optimal Operational Planning for PV-Wind-Diesel-Battery Microgrid. The authors used a commercial solver called CPLEX in a personal computer to run the algorithms.

Another paper - [14], uses MILP to solve economic energy dispatch in buildings and microgrids. The problem formulation is complex and involves several parameters in a hospital environment.

In this paper, a simple economic energy dispatch optimizer system is proposed and it's called "Optimizer in a Box", running in a personal computer and low cost embedded systems.

The organization of the paper is: In section II a brief review of the OPC UA Architecture is presented. In section III a Dispatch Optimization in Small microgrids and Green Data Centers is discussed and a communication model based in OPC UA and a microgrid Optimization Problem is presented. In section IV several simulations scenarios are evaluated and the results of the optimization solution are presented. In section $\mathrm{V}$ the conclusions and future works are discussed.

\section{OPC UA ARCHITECTURE}

OPC-UA is a specification from the OPC Foundation, as a middleware for RPC (Remote Procedures Call), that operates in the client/server paradigm. This specification is standardized by IEC 62541 . This standard does not provide one client and server API, but the OPC Foundation offers to 
its members, client SDKs, open basic server, and ANSI C language, based on the standard. Many solution providers sell SDKs that provide friendly APIs (stack wrappers) on several platforms (like $\mathrm{C}, \mathrm{C}++, \mathrm{C} \#$ and Java) for development of client and server applications. Figure 1 shows the architecture of standard OPC-UA.

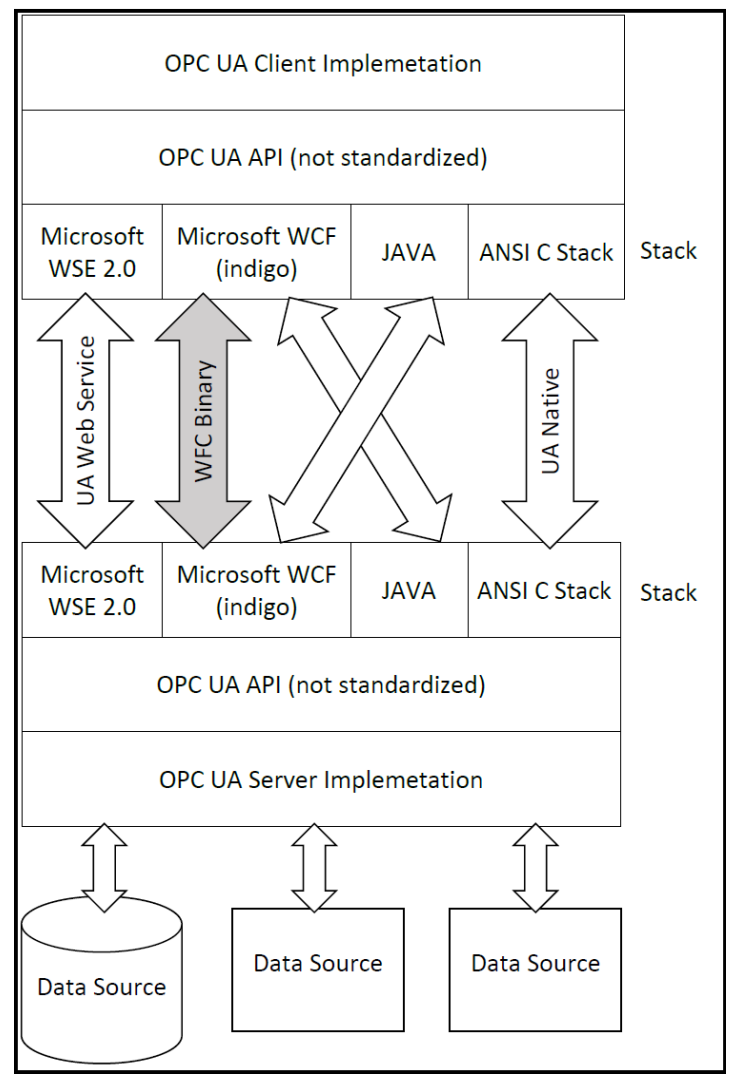

Figure 1. OPC-UA architecture [3]

The client application uses the API to send service requests. The messages exchanged between the client and server can use XML/SOAP encapsulation in the HTTP(S) for Web Service, favoring interoperability with enterprise systems such as ERP. However, applications using Web Services or even the DPWS specification (Device Profile for Web Service) that allow the implementation of service-oriented architecture in embedded systems with reduced processing power, have performance problems due to the complexity of SOAP messages, as demonstrated in [2], particularly where the establishment of secure channels is required. Thus, the messages exchanged between client and server can be encapsulated over a new binary protocol called "TCP-UA" or "UA Native Binary". This new approach enables message mapping directly in a compressed package over TCP.

In both mappings (XML/SOAP and UA Native) it is possible to connect a device on the shop floor or in a microgrid bay, directly through an OPC-UA client, with high performance and security, cross firewalls, as shown in figure 2.

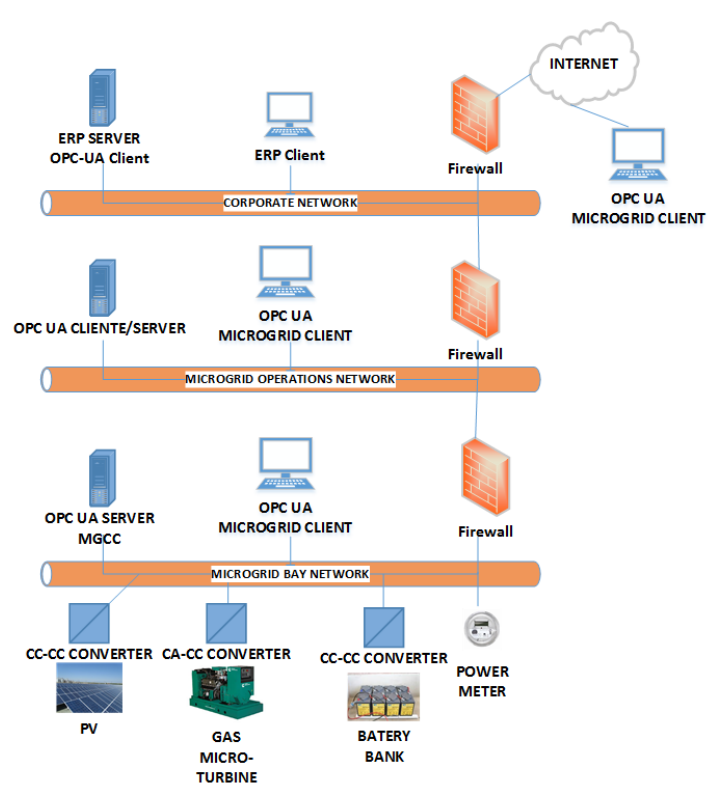

Figure 2. OPC-UA network

\subsection{OPC-UA Features}

OPC-UA extended the functionality of traditional OPC. The main new features are self-discovery features, event subscription for monitoring items and security services. The self-discovery and self-description features provide plug and play capability to intelligent electronic devices (IEDs) in a bay substation, for example. The event services provide efficient and easy monitoring of critical changes in the IEDs parameters, like current and voltage surge alarms. The security services mitigate cybernetic attacks to the systems.

\subsubsection{Discovery Services}

OPC-UA offers self-discovery capabilities, allowing customers automatic detection of available servers on local network or multicast groups, allowing plug-and-play features, similar to those found in DPWS technology [3]. Figure 3 shows how the OPC-UA Discovery service operates.

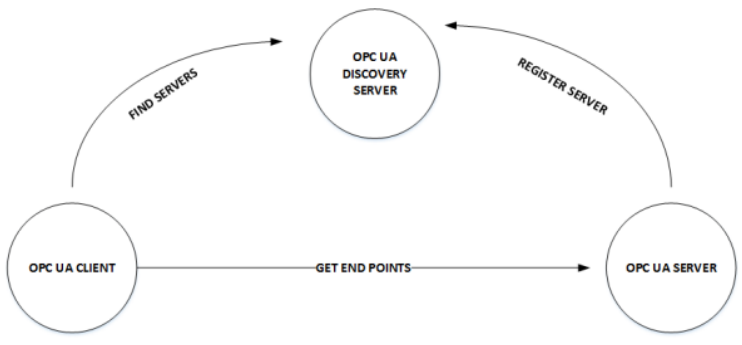

Figure 3. OPC-UA Discovery service

As shown in Figure 3, the client sends an event message to a discovery server that contains the list of available registered servers. The discovery server returns to the client a single server identifier, called an "Endpoint", from which the client can establish a session.

\subsubsection{Event Subscription Service}

The subscription service is available on the server to the client. The client sends an event subscription request to the server with the list of monitored items. In well-defined time intervals (and negotiated with the client) the server samples the value of variables from monitored items and sends 
notifications to a queue so that they are notified to the client, as shown in figure 4 .

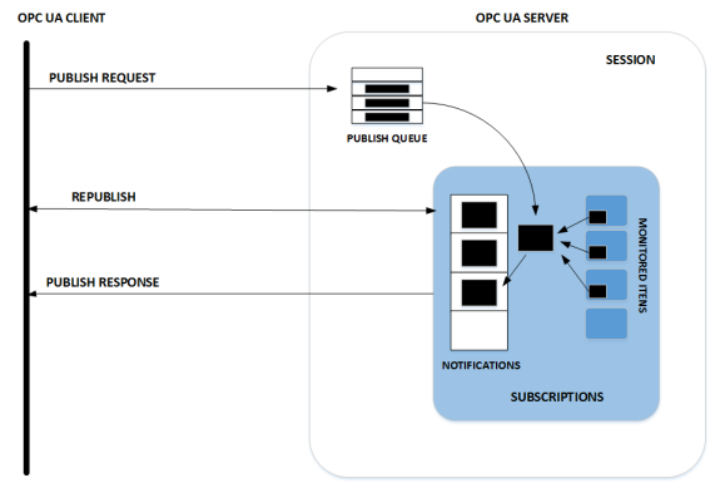

Figure 4. Event Subscription

\subsubsection{Security Services}

The OPC-UA architecture natively adds security features, letting the client application choose the security policy available on the server, such as symmetric and asymmetric encryption, to provide end-to-end security, mitigating among others the "man in the middle" attack [4]. The security mechanisms of OPC-UA are the same as used in Web Services technology and well validated in enterprise applications. Fig. 5 illustrates the OPC-UA security model.

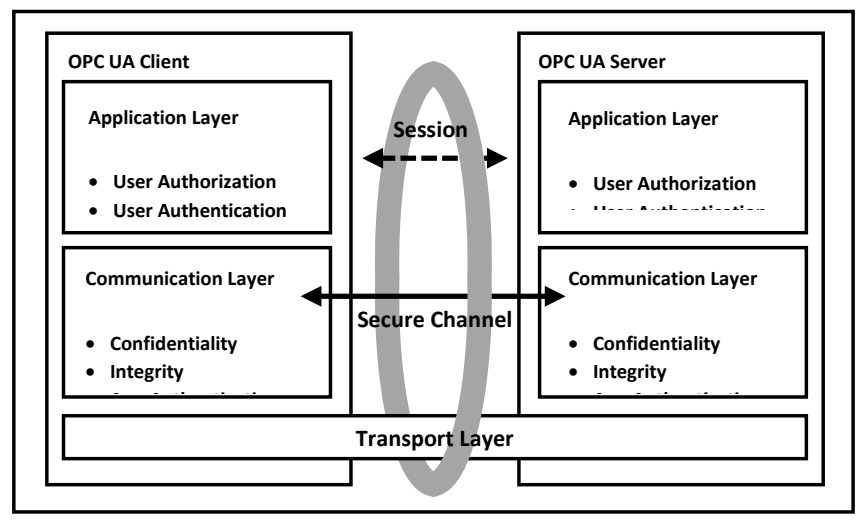

Figure 5. OPC-UA security model

\section{DISPATCH OPTIMIZATION IN SMALL MICROGRIDS AND GREEN DATACENTERS}

A microgrid is a local energy network involving energy sources and energy storage systems. These systems must include local control features. They should also be able to detect main network failures to disconnect and generate energy autonomously for local loads in an operating mode called "islanded" [5].

With the growth of cloud and data processing, large information technology facilities operating in network environment of massively distributed computers are proven to be one of the major consumers of electricity, operating $24 \times 7$, requiring an uninterrupted power supply. Energy consumption growth in these facilities has motivated the creation of the so-called "Green Data centers", which according to [1] are data centers that adopt techniques of efficient and environmentally friendly energy consumption. For that, they employ micro-generation arrangements using alternative non-fossil energy sources such as photovoltaic, wind and others sources of energy. Green data centers are characterized by the incorporation of non-linear electrical loads such as computers and network equipment, which can be powered from DC power networks. Moreover, a significant component of the load in these facilities are air conditioning systems, powered by alternating current. Green data centers' energy supply systems can strongly benefit from the microgrid concept.

In order to meet the requirements of energy and economic efficiency of microgrids, a need arises for centralized management architecture, employing a microgrid control node called microgrid Central Controller - MGCC [6]. Fig. 6 shows the architecture of a microgrid employing a MGCC.

In this scenario, the MGCC needs to be data network connected with other microgrid devices and, in the case of distributed generation, with the energy supply systems. In both cases, the performance and safety requirements to SmartGrids communication systems must be observed. The MGCC performs the following functions:

- Establishes and manages the best technical and economic policy for dispatch control and management on the demand side manner of the microgrid generation;

- Receives parameters, processes and sends the set points, to the microgrid energy resources devices.

The scope of this proposal is focused on a small islandedcapable dc microgrid, with a photovoltaic generation system, employing a MGCC. The communication infrastructure uses the OPC-UA as middleware communication, between the MGCC and all other microgrid devices.

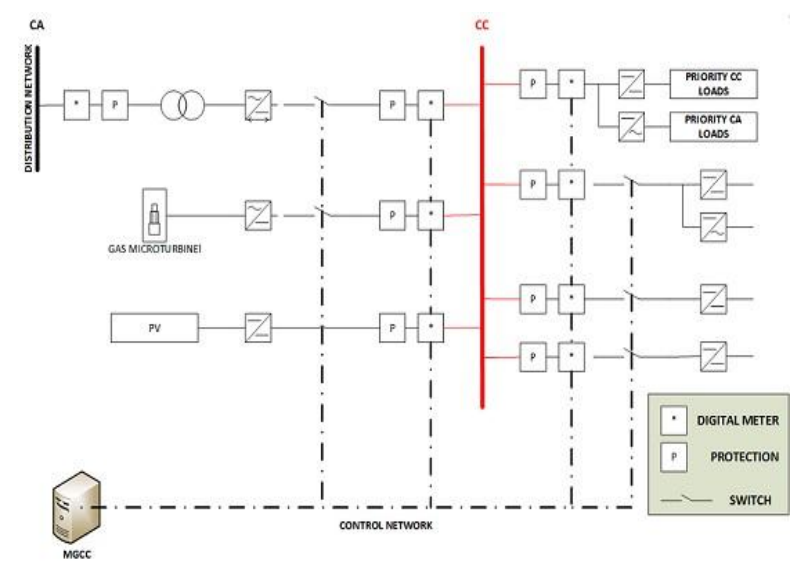

Figure 6. Considered microgrid with MGCC Architecture

\subsection{OPC-UA Communications Infrastructure}

SmartGrids must present interoperability and security requirements in order to allow their remote monitoring and management, often using the Internet infrastructure in micro distributed generation arrangements.

In this perspective, OPC-UA enables compliance with the microgrids requirements, such as robust and fault tolerant operation, as well as two-way, end-to-end secure communication infrastructure. OPC-UA provides a natively service-oriented architecture paradigm. This feature favors the integration of microgrids to the corporate management system, such as ERPs (Enterprise Resources Planning) 
applications, with support for supervision and control tools such as SCADA (Supervisory, Control and Data Acquisition).

As shown in Fig. 6, the model presupposes the use of intelligent inverters and converters, supporting OPC-UA or legacy protocol through the use of gateways that convert them to OPC-UA. The second option was adopted in this proposal. The use of OPC-UA security features is assumed to create secure channels between MGCC (OPC-UA client) and the microgrid devices (OPC-UA servers) employing asymmetric cryptography of Public Key Infrastructure (PKI).

In this model the MGCC is a simple personal computer that will run an OPC-UA client application responsible for reading at regular times (polling) each device data (server) to perform an energy flow dispatch optimization problem instance. The variables of interest are:

- Pmax: maximum power that can be generated at the time of reading by the generating unit.

- PTO: state variable that indicates the operating condition of the generating unit. If PTO $=1$ the unit is generating energy normally. If PTO $=0$, the unit has failed (in this case Pmax should be considered zero for the optimizer). If PTO $=2$ it means that unit is in the loading ramp in the case of batteries or starting ramp in case of diesel generator or gas micro turbines. In the latter two cases, Pmax should be considered zero by the optimizer.

- C: generation costs of the units. The generation costs will be provided by a specific server that will maintain the seasonal billing tables from the utility, fuel prices, maintenance cost tables of solar panels and battery banks.

- $\quad$ PL: Power loads. A power meter (server) could provide the current power load demand. When the battery banks are in the loading ramp its power is negative, that is, its absolute value must be added to the power consumed by the load group for optimizer purposes.

- Pref: reference power to configure the inverters and converters after each round of the optimization algorithm.

- $\quad$ SoC: State of Charge for every battery bank, provided by a commercial BMS (Battery Management System) attached to the battery server. Constant for each optimization round.

- SoH: State of Health, provided by BMS. Constant for each optimization round.

The MGCC subscribes to the OPC-UA event service of each server associated with the generating units, so that the MGCC should be notified at every change in the value of PTO. At such events, a new reading of Pmax and PTO must be done automatically, regardless of the polling time, and a new optimization instance must be immediately executed. The MGCC is responsible for the following activities:

- $\quad$ Reading of data from the server;

- Registration and monitoring items;

- $\quad$ Running the optimizer;

- $\quad$ Rotating the battery banks based on SoH parameter to optimize the life time.
- $\quad$ Setting the inverters and converters to the power values resulting from the optimizer by writing the Pref register (reference power);

- Generating tabular and charts reports.

The OPC-UA servers will be connected to the power inverters connected to the utility and the diesel generator (or gas micro turbine), inverters connected to the battery bank and the PV generators. They are responsible for sending Pmax and PTO.

The servers can be deployed on embedded devices connected directly to the power inverters and converters. In addition, the servers must provide an OPC-UA event service, every time that the value of the PTO value is changed. The OPCUA server connected to the power meters sends the power consumed by the loads.

\subsection{The Microgrid Optimization Problem}

As previously discussed this proposal is applied to a small DC microgrid associated with a data center with the use of photovoltaic sources. The optimization strategy is focused on minimizing economic costs of dispatching electrical power in the microgrid with fault tolerance (in a $24 \times 7$ operation mode). The control strategies of the converters and inverters are not considered here.

In this scenario the microgrid optimization problem consists of minimizing the cost of feeding the electric charges. The objective function (OF) will take the following criteria:

- Generation costs of the diesel generator (fuel value). For an initial simplification maintenance costs due to the start / stop of the generator will not be considered;

- Costs/allowances/remuneration for consumption or generation to the dealership (the value of costs/allowances/compensation agreed upon with the same depending on seasonal rates and peak times will be considered).

The system constraints take the following criteria:

- Instant load demand (measured) of DC and AC loads;

- Charging ramp of the battery bank;

- Generation capacity of the units (AC network, diesel generator, battery banks and PV);

- For diesel unit the starting ramp generator should be considered. From the moment it's in the regime, its generation capacity will be considered by the manager as nominal. The generated power can be measured by digital equipment;

- $\quad$ For PV unit the instantaneous generated power will be provided by the DC-DC converter connected to it.

- $\quad$ SoC of every battery bank.

The optimization problem was mathematically modeled as a linear programming problem described by the equations below:

Decision variables:

- P1 - Power being supplied by the AC line concessionary; 
- P2 - Power being supplied by the diesel generator;

- $\quad$ P3 - Power being supplied by the photovoltaic panels;

- $\quad$ P4 - Power being supplied by the battery bank 1;

- $\quad$ P5 - Power being supplied by the battery bank 2;

Objective Function:

$$
\text { O.F.: Minimize }{ }^{X} C_{i} \times P_{i} \quad i=1 \ldots 5
$$

Where $\mathrm{Ci}$ the economic costs of generating units. For batteries bank the economic cost was penalized for $\mathrm{SoH}$ and SoC.

This approach extends the life cycle. The penalized battery costs are:

$$
C_{i} \times S o C^{-1} \times S o H^{-1}
$$

Subjected to:

$$
\begin{aligned}
& P 1 \leq P 1 \max \\
& P 2 \leq P 2 \max \\
& P 3 \leq P 3 \max \\
& P 4 \leq P 4 \max \\
& P 5 \leq P 5 \max \\
& \operatorname{SoC}_{i}>\operatorname{Min} ; \quad i=b_{1} t_{1}, b_{2} t_{2} \ldots b t_{n} \\
& \mathrm{SoC}_{i}<\operatorname{Max} ; \quad i=\mathrm{bat}_{1}, \mathrm{bat}_{2} \ldots \mathrm{bat}_{n} \\
& (P 1+P 2+P 3+P 4+P 5) \geq P R L \\
& P i \geq 0 ; \quad i=1 \ldots 5
\end{aligned}
$$

Where:

- Pmax: nominal power of the unit

- PRL: total power load. When battery was in charge, the power is considered in the PRL.

- $\quad$ SoC: Percentage State of charge for batteries

- $\quad$ Min and Max are the SoC limits for bank batteries

To solve the above problem, a simple algorithm and the programming tool called GLPK API - [7] were used because it is effective for the proposed issue and distributed in a free and open source license. Therewith, we can generate $\mathrm{C}$ code in the various instances of the problem at regular intervals of time, reading system variables from the data available in the converter units and existing energy meters to optimally set up the dispatch of the energy to the loads in the microgrid.

The problem is simple, and can be solved in closed form in polynomial time through the simplex algorithm [8] with a small instance (few generating units).

The simple algorithm used to solve the problem is showed above.
Result: Power Reference for Generation Units initialization;

for Every hour of day do

Read decisions variables, restrictions, SoH for every power unit; Read load demands;

if $\mathrm{SoH} 1 \leq \mathrm{SoH} 2$ then

Multiply Bat1 cost; else

Multiply Bat2 cost;

end

Run Optimizer

Configure PReffor every generation Units

end

Algorithm 1: Optimized Load Balance Algorithm

\section{RESULTS}

To evaluate the problem modeling and solution a set of simulations were executed. Three scenarios were evaluated:

- No fault in utility;

- $\quad$ Off-Peak fault (diurnal - PV contribution);

- $\quad$ On-peak fault (nocturnal - No PV contribution);

- Optimizer OFF (all costs are equal);

Figure 7 show Pmax for every power unit and load demands for a January day (not holiday) in Florianópolis - Southern Brazil.

Figure 8 shows the normalized costs of each power unit. This costs are based in economic costs of the units. The utility power costs are based on the seasonal table of costs of a power distribution utility in the Florianópolis city. The CHP (Diesel generator) costs are based on the fuel price. The costs of battery banks and solar panels were considered as small values because the maintenance costs were not considered. When the battery bank is in the charge ramp, the costs are configured with the maximum value.

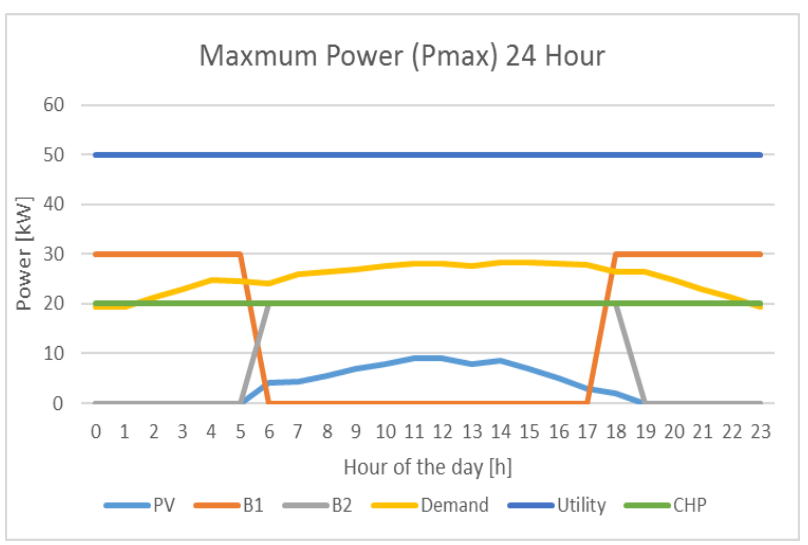

Figure 7. Maximum Power (Pmax) - 24 hour 


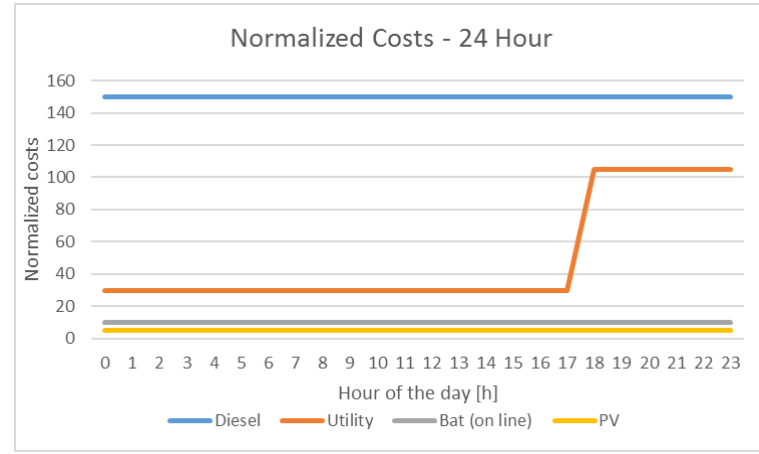

Figure 8. Normalized costs - 24 hour

\subsection{No Fault Scenario}

In this scenario, the power utility was operated in every hour of the day. Figure 9 shows the results for the optimized load balance.

In all cases the SoC (State of Charge) decrease was considered linear.

For battery bank 1 SoC begins at $40 \%$. For battery bank 2 SoC begins at $80 \%$. For SoC less than $20 \%$ the battery was configured to charge ramp and Pmax was set to zero. As presented in figure 8 , the utility provides the complementary power along the commercial timeframe (7:00 AM to 6:00 PM). Outside this time range, the bank batteries and PV, supply the power for the grid with low cost.

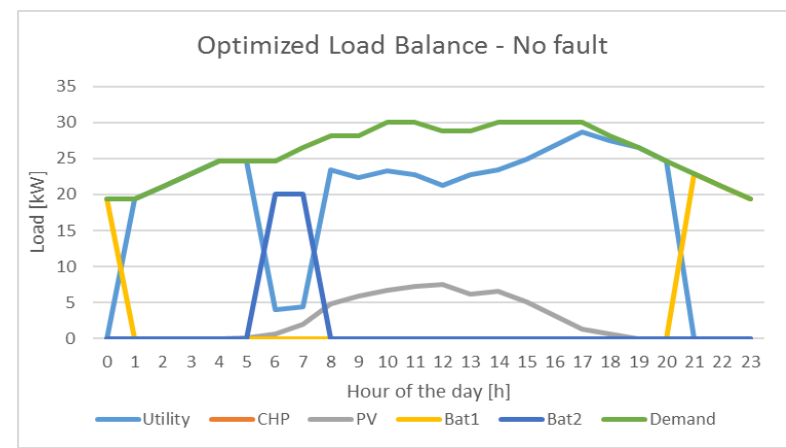

Figure 9. Optimized Load Balance - No fault

\subsection{Fault scenario off-peak - Diurnal Operation}

In this scenario, a utility fault is simulated in off-peak time. Figure 10 shows the optimized load balance.

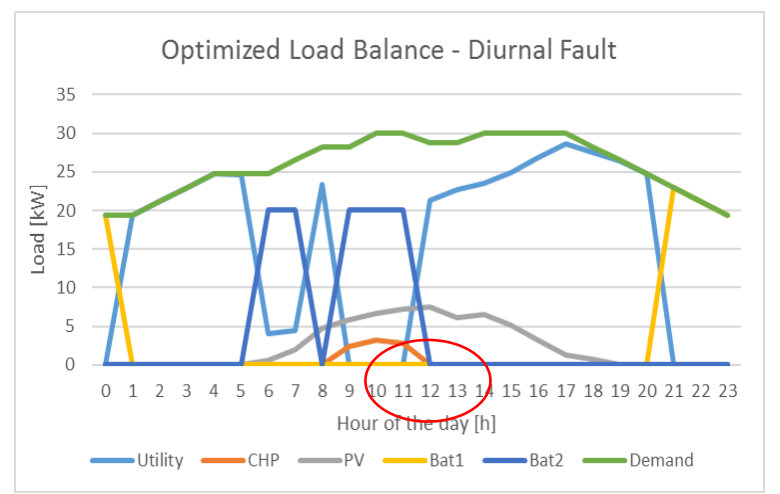

Figure 10. Optimized Load Balance - off-peak time with fault
Figure 10 shows the need to activate the CHP generator to meet load demands.

\subsection{Fault scenario on-peak - Nocturnal Operation}

In this scenario a utility fault at peak time is simulated. In this time range, there is no PV contribution to meet power demands. The figure 11 shows the optimized load balance. Again, the diesel generator was needed.

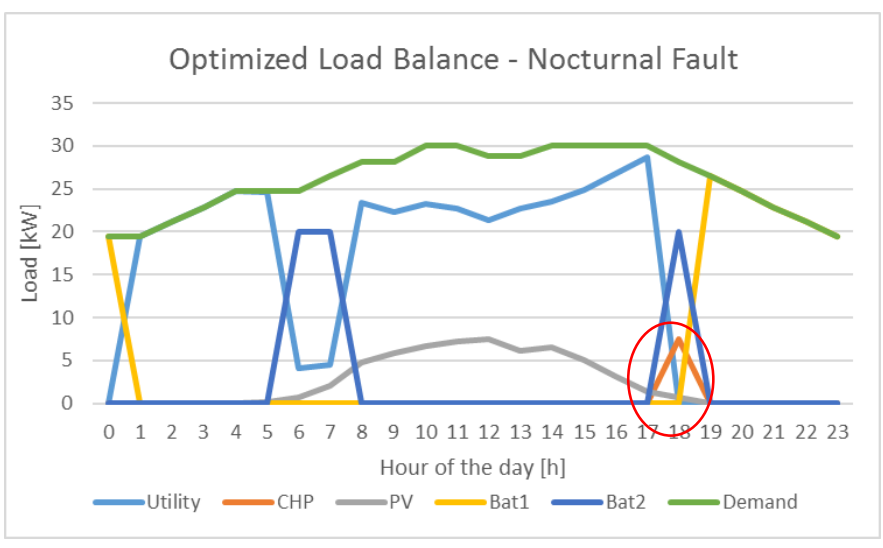

Figure 11. Optimized Load Balance - on-peak time with fault

\subsection{Conclusions}

After these three scenarios were tested, it was found that the optimizer's results were coherent for all cases. The load demands were supplied by generation units and the power setup decreased the economic cost.

The computational time is very low (less than $0.5 \mathrm{~ms}$ ) using an Intel Core I7 processor with five generation units and one power meter for load demands. The results were indicated that the optimizer's interval can be less than 1 hour (30 minutes, 15 minutes or below). The number of units can be expanded.

The economic efficiency of the optimizer is shown in figure 12. In this figure the value of the objective function with no optimizer and two scenarios (no fault and Diurnal Peak-time) was evaluated. For scale reasons, the peak-time instances were not shown.

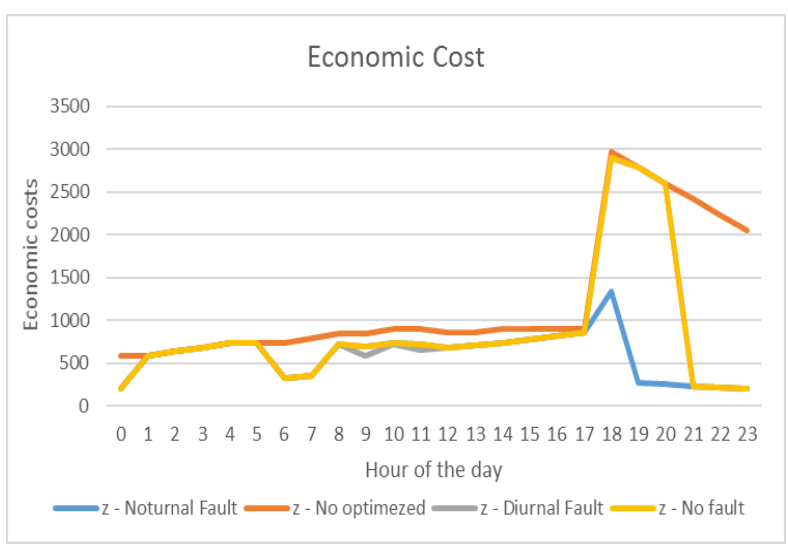

Figure 12. Objective Function

Figure 12 shows that the cost of the non-optimized case is greater than that of other cases, as expected, even in the case of an utility fault (CHP was used). For case "Nocturnal Fault" the accumulated ( 24 hour) economic gain is $107,40 \%$. 
For case "Diurnal Fault" it is $43,67 \%$. For case "No fault" it is $42,37 \%$.

\section{CONCLUSIONS AND OUTLOOK}

In this paper a simple optimizer for economic minimization problem of power dispatch in small microgrid was presented. The problem was solved with a simple algorithm and modeled as a linear programming solved by open source package called GLPK Solve. The communication infrastructure used in this paper is based on the new OPCUA protocol, easy to use and compatible with Service Oriented Architecture. The simulation results point to the efficient use of renewable power sources, minimizing the costs to meet load demands. In all tested cases, the value of objective function of problem is less when the optimizer is turn-off. The implementation of this system has a low cost and is easy to operate. To integrate this system with a power utility concessionary, OPC-UA offer security features over the Internet, with large interoperation resources.

In future work a promising protocol for IIoT called "MQTT" (Message Queue Telemetry Transport) will be implemented in the microgrid scenarios and its performance will be compared to the OPC UA's.

\section{ACKNOWLEDGMENT}

This work is partially sponsored by CNPq/Brazil (National Council for Scientific and Technological Development Brazil). Proc. Numbers: 487918/2013-4, 467015/2014-7 and 310222/2014-1.

\section{REFERENCES}

[1] Control, Automation and Robotics- ICINCO-RA. 2009.

[2] Elsayed, Ahmed T., Ahmed A. Mohamed, and Osama A. Mohammed. DC microgrids and distribution systems: An overview. Electric Power Systems Research 119 (2015): 407-417.

[3] Liaria, Alvaro, et al. Survey on microgrids: unplanned islanding and related inverter control techniques. Renewable Energy 36.8 (2011): 2052-2061.

[4] Thorncraft, Stuart R., Hugh R. Outhred, and David J. Clements. Evaluation of open-source LP optimization codes in solving electricity spot market optimization problems. Mini-Euro Conference on Operation Research Models and Methods in the Energy Sector. 2006.

[5] Klee, Victor, and George J. Minty. How good is the simplex algorithm. No. TR-22. WASHINGTON UNIV SEATTLE DEPT OF MATHEMATICS,1970.

[6] Industry Agenda. Industrial Internet of Things: Unleashing the Potential of Connected Products and Services 1970.
[7] Mackiewicz, R. E. "Overview of IEC 61850 and Benefits." Power Systems Conference and Exposition, 2006. PSCE'06. 2006 IEEE PES. IEEE, 2006.

[8] Suic, Stjepan. "Optimizing OPC UA middleware performance for energy automation applications." Energy (2014).

[9] Pedersen, Anders Bro, et al. "Facilitating a generic communication interface to distributed energy resources: Mapping IEC 61850 to RESTful services.' Smart Grid Communications (SmartGridComm), 2010 First IEEE International Conference on. IEEE, 2010.

[10] Moshi, Godfrey Gladson, Cristian Bovo, and Alberto Berizzi. "Optimal operational planning for PV-WindDiesel-battery microgrid." PowerTech, 2015 IEEE Eindhoven. IEEE, 2015.

[11] Stluka, Petr, Datta Godbole, and Tariq Samad. "Energy management for buildings and microgrids." Decision and Control and European Control Conference (CDCECC), 2011 50th IEEE Conference on. IEEE, 2011.

\section{AUTHOR PROFILE}

R. A. Dias: Bachelor's at Electric Engineering from Federal University of Santa Catarina (1988), Master's at Mechanical Engineering from Federal University of Santa Catarina (1996) and doctorate at Electric Engineering from Federal University of Santa Catarina (2004). Has experience in Computer Science, focusing on Telecomputing, acting on the following subjects: web services and distributed systems.

G. C. Gomes: technologist in Industrial Mechatronics (2012), Master's of Mechanical Engineering from the Federal University of Santa Catarina - UFSC specialized in the area of metrology and instrumentation. Professor at the Technical Course in Industrial Automation at SENAI-SC, Florianópolis unit and external researcher at the Group of Embedded and Distributed Systems at Federal Institute of Santa Catarina. Has experience in the fields of mechatronics, embedded and distributed systems and automation - with an emphasis on instrumentation and automation tests using Labview platform.

M. L. Heldwein: holds a degree (1997) and MA (1999) in Electrical Engineering from UFSC and doctorate (2007) by the ETH Zurich. He is currently an assistant professor in the Department of Electrical Engineering of UFSC. From 1999 to 2001 he worked as a research assistant in the Power Electronics Institute (INEP). From 2001 to 2003 he was an engineer of R \& D at Informat, working on sources of project with Emerson Network Power. From 2003 to 2008 he worked at the ETH Zurich in Power Electronics area. He is a Senior Member of the Institute of Electrical and Electronics Engineers (IEEE) and a member of the Association of Power Electronics (SOBRAEP). 